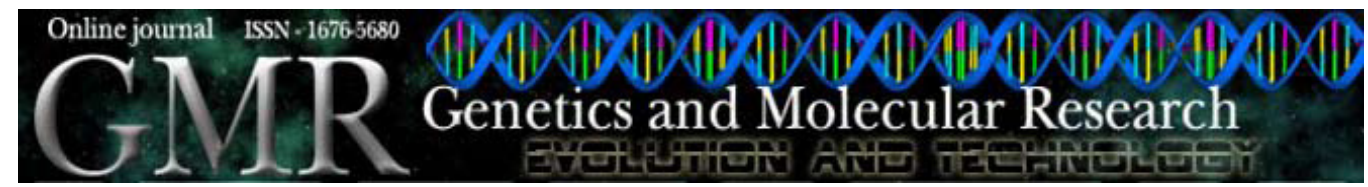

Review

\title{
Intrinsically bent DNA in replication origins and gene promoters
}

\author{
F. Gimenes ${ }^{1}$, K.I. Takeda ${ }^{1}$, A. Fiorini ${ }^{2}$, F.S. Gouveia ${ }^{1}$ and M.A. Fernandez ${ }^{1}$ \\ ${ }^{1}$ Departamento de Biologia Celular e Genética, \\ Universidade Estadual de Maringá, Maringá, PR, Brasil \\ ${ }^{2}$ Centro Universitário de Maringá, CESUMAR, Maringá, PR, Brasil \\ Corresponding author: M.A. Fernandez \\ E-mail: aparecidafernandez@gmail.com
}

Genet. Mol. Res. 7 (2): 549-558 (2008)

Received March 18, 2008

Accepted June 6, 2008

Published June 24,2008

\begin{abstract}
Intrinsically bent DNA is an alternative conformation of the DNA molecule caused by the presence of dA/dT tracts, 2 to 6 bp long, in a helical turn phase DNA or with multiple intervals of 10 to $11 \mathrm{bp}$. Other than flexibility, intrinsic bending sites induce DNA curvature in particular chromosome regions such as replication origins and promoters. Intrinsically bent DNA sites are important in initiating DNA replication, and are sometimes found near to regions associated with the nuclear matrix. Many methods have been developed to localize bent sites, for example, circular permutation, computational analysis, and atomic force microscopy. This review discusses intrinsically bent DNA sites associated with replication origins and gene promoter regions in prokaryote and eukaryote cells. We also describe methods for identifying bent DNA sites for circular permutation and computational analysis.
\end{abstract}

Key words: Intrinsically bent DNA; Replication origin; Promoters; Prokaryotes; Eukaryotes 


\section{INTRODUCTION}

The DNA molecule is not always found in a linear structure inside the nucleus. The DNA sequence may assume three-dimensional conformations depending on its sequence, which may include cruciform shapes, loops, and curves (Calladine et al., 2004).

The most important characteristic responsible for intrinsic curvature or bent DNA is the presence of adenine/thymine-rich tracts periodically spaced along the DNA molecule with repetitions or multiples of $10 \mathrm{bp}$ (Haran and Crothers, 1989). A probable explanation for the formation of this curvature is the interaction of the $\mathrm{A} / \mathrm{T}$ base pairs, which allows the formation of a cross link between one oxygen atom of thymine and one nitrogen atom of adenine in two consecutive pairs. This cross link connection allows base pairs with consecutive A/T tracts to maintain a closed structure (Calladine et al., 2004) and promotes a natural curvature of the helix. Researchers have described curvatures in DNA with other combinations of these tracts (Bolshoy et al., 1991) and also some DNA sequences lacking A/T tracts (Fujimura, 1988).

Bent DNA sites are involved in specific regions of the genome where the DNA and proteins interact, with outcomes such as gene recombination (Iarovaia et al., 2006; Barbosa et al., 2008), replication origins (Altman and Fanning, 2004; Fiorini et al., 2006a, Balani et al., 2006; Gimenes et al., 2008), transcription (Marilley and Pasero, 1996; Bash et al., 2001; Fiorini et al., 2001; Reis et al., 2004; Gouveia et al., 2008), nucleosome compaction (Virstedt et al., 2004), fragile sites (Palin et al., 1998), and tandem repeated sequences (Pasero et al., 1993). Intrinsically bent DNA is localized near or at scaffold/matrix attachment regions (S/ MARs; Brylawski et al., 2000; Fiorini et al., 2006b), thus demonstrating that DNA conformation is important for the functioning of biological processes.

Intrinsically bent DNA sites can be detected by several assays, including electrophoretic behavior (Fiorini et al., 2006b), computational analysis, and force atomic microscopy (Marilley et al., 2007). The detection of curvature sites near or within chromosome regions allows for the establishment of a relationship between structure and function. This study reviews the main detection methods for identifying bent DNA sites and the occurrence of these structures associated with replication origins and promoters in prokaryotic and eukaryotic organisms.

\section{DETECTION OF BENT DNA}

\section{Computational analysis}

Structural bends in a DNA sequence can be located by computational analysis using specific software that displays the results in both graphic and 3-D modeling formats. These analyses support electrophoretic behavior analysis, as well as other assays for bend detection.

The dinucleotide wedge model of Trifonov and Sussman (1980) suggests that the curvature occurs due to alterations in the AA steps. Another model, the junction model, proposed by Levene and Crothers (1983), suggests that intrinsically bent DNA sites are only located at the junction between the A-tracts and the surrounding sequence. A combination of these models, the mixed sequence model, indicates that DNA curvature is caused by multiple slight bends between adjacent bases outside the A-tracts (Young and Beveridge, 1998). 
Curvature peaks and the 2-D projection of the 3-D trajectory from specific fragments can be obtained from computational programs such as Map15a and 3d15m1 (Pasero et al., 1993; Fiorini et al., 2001). These programs use the Eckdahl and Anderson algorithm (1987) and the helical parameters of Bolshoy et al. (1991), Pasero et al. (1993), and Marilley and Pasero (1996). The ENDS ratio parameter (ratio of the axis outline of the helix to the smallest distance between the extremities of the fragment) is generally used for putative bend detection (Bolshoy et al., 1991; Pasero et al., 1993). The window width and the base pair window can be altered according to the fragment weight.

An example of this application is shown in Figure 1A and B, for a hypothetical segment of $670 \mathrm{bp}$, using a 120-bp window width and a 10-bp step. Figure 1A shows two main peaks of bent sites ( $b 1$ and $b 2)$ with ENDS ratio values of 1.23 and 1.45 , respectively. Values greater than or equal to 1.10 signify reduced mobility indicative of bent DNA (Milot et al., 1992). The 2-D trajectory showing the $\Delta \mathrm{G}$ values (which predict the stability of the DNA duplex) was performed for this fragment using the 3D15m1 software (Figure 1B). Through this analysis, it is possible to verify that the variation of free energy around the bend regions is negative compared to straight sequences.

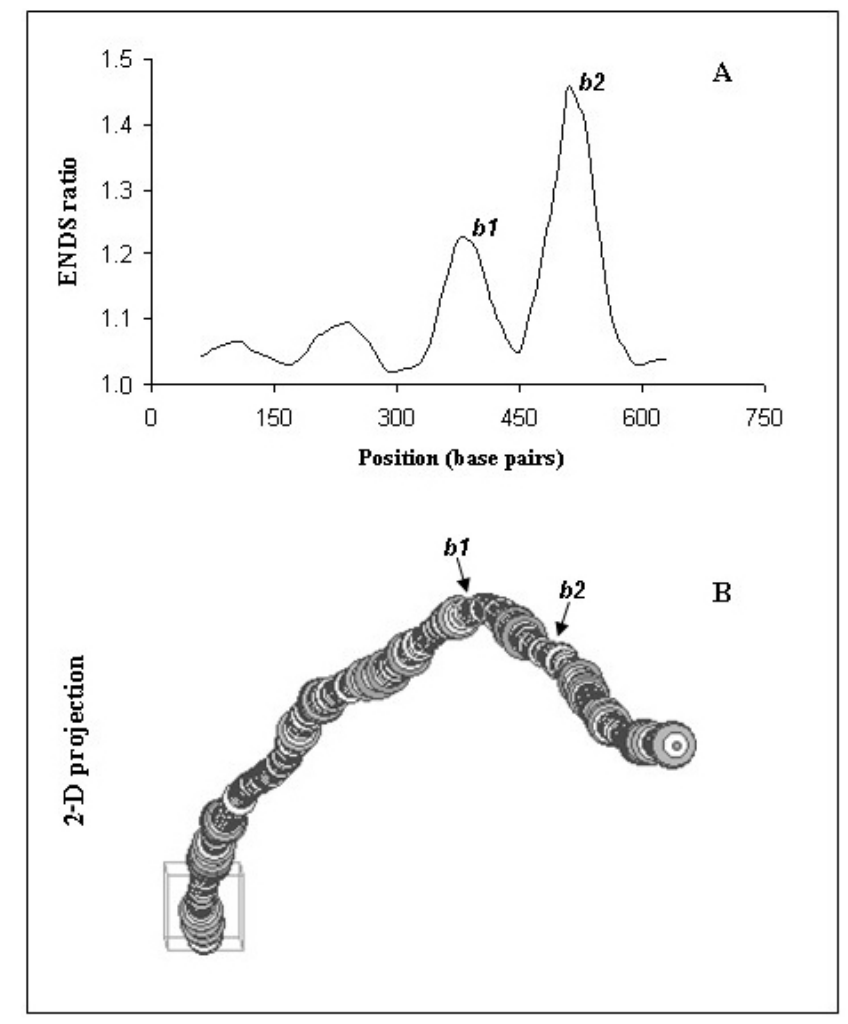

Figure 1. Computational analysis of a hypothetical 670-bp fragment with two bending sites. A. ENDS ratio analysis showing the peaks of major curvature with values greater than or equal to 1.10. B. 2-D projection of the 3-D trajectory of the hypothetical fragment. Circles of different sizes and black to gray intensities represent the Gibbs free energy variation $(\Delta \mathrm{G})$ along the sequence, which predicts the double helix stability. 


\section{Circular permutation}

This assay is used to determine whether bent DNA sites promote curvature in a distinct sequence context and to determine the bending-center sites on permuted fragments $(\mathrm{Wu}$ and Crothers, 1984; Kim et al., 1989).

The fragment containing the bent site is cloned into the center of a duplicated polylinker of pBendBlue vector (Sperbeck and Wistow, 1998). The vector containing the insert is cleaved by restriction enzymes, with cleavages occurring once in each polylinker, and the resulting fragments are electrophoresed on polyacrylamide gels at $4^{\circ} \mathrm{C}$ without any intercalating agent such as ethidium bromide (Figure $2 \mathrm{~A}-\mathrm{C}$ ).

A
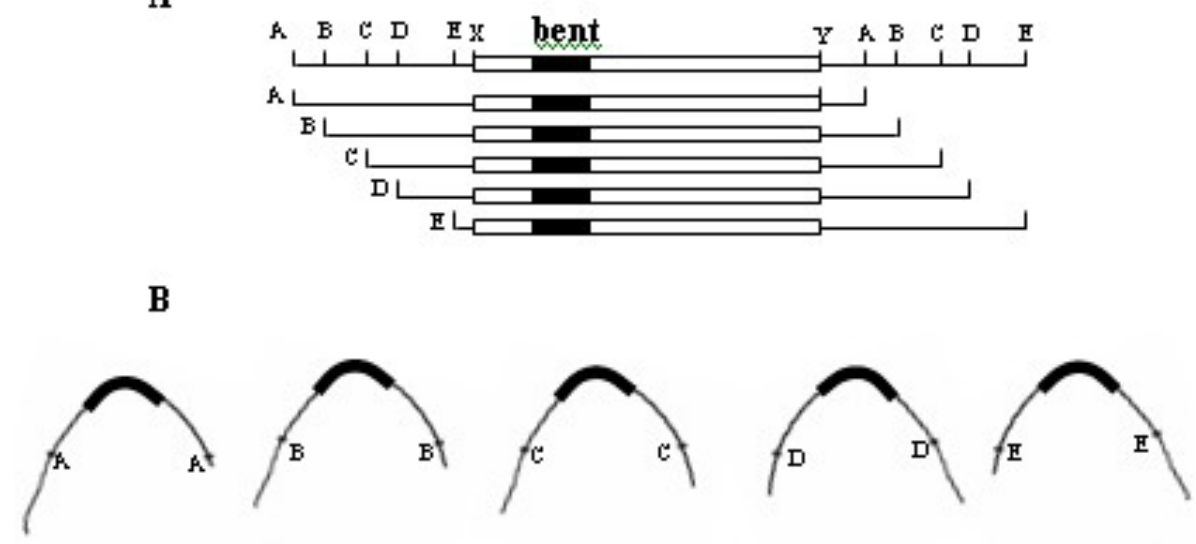

C

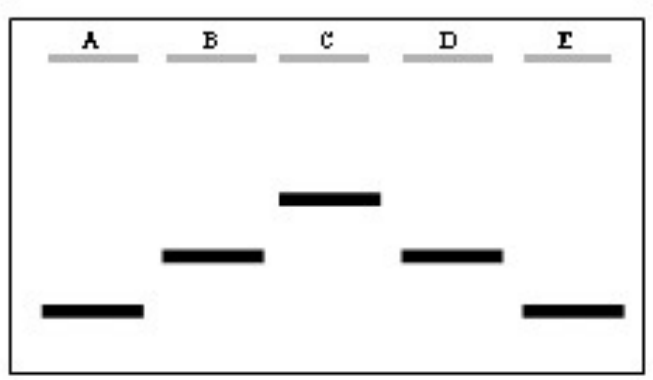

Figure 2. A schematic representation of a DNA fragment circularly permuted. A. The open bars represent the analyzed fragment to be inserted in the middle of the duplicated polylinker of the permutation plasmid, like pBendBlue. The closed bars represent the bent DNA site to be mapped. Cleavages with restriction enzymes A, B, $\mathrm{C}, \mathrm{D}$, and E produce a fragment with the same weight, but with the bent site permuted. The insert is cloned between the restriction sites $\mathrm{X}$ and $\mathrm{Y}$. B. The 2-D projection of the circularly permuted fragments. C. Pattern of permuted fragment migration on polyacrylamide gel electrophoresis. 
The cleavage generates a set of fragments of identical length, but with the bent site at different positions relative to the fragment ends (Figure 2A and B). The fragment with the bending site in the center shows the maximum retardation on the polyacrylamide gel compared to fragments where the bending site is at the end (Figure 2C).

The mobility pattern of each fragment can be obtained through the calculation of the $R$-value (ratio of apparent/real fragment size observed in each gel system). $R$-values between 0.90 and 1.09 indicate no alterations in mobility, those greater than or equal to 1.10 indicate reduced mobility, and those less than 0.90 indicate fast mobility (Fiorini et al., 2006a).

\section{BENT DNA SITES IN PROKARYOTIC REPLICATION ORIGIN}

Prokaryotic cells contain circular chromosomes where DNA replication starts from a single origin; this contributes to a fast replication process (Wang and Sugden, 2005; Robinson and Bell, 2005). The DNA sequence motifs, such as DnaA box, protein-binding, and those that direct the opening of the DNA in the bacterial replication process (for a review, see Messer, 2002), as well as other motifs within these, may be associated with intrinsic curvatures.

The analyzed molecular structure of DnaA protein in bacterial cells reveals high affinity when curved DNA regions exhibit negative supercoiling (Remus et al., 2004; Clarey et al., 2006; Erzberger et al., 2006). Curved DNA and negative supercoiling are necessary for the creation of the single structure at oriC that allows the cell to properly initiate DNA replication. Additionally, it has been shown that the SeqA protein in bacterium, which represses DNA replication activity, promotes helical changes in DNA structure that causes positive supercoiling of the segment (Klungsoyr and Skarstad, 2004).

The Xylella fastidiosa chromosome exhibits rich phased AT tracts, CAnT motifs, and five putative DnaA box sequences (R1-R5), as well as a box of 24 nucleotides with 8 ACC repetitions (Monteiro et al., 2001). These regions are important for both protein-binding and the opening of the DNA during the bacterial replication process. Gimenes et al. (2008) have reported three bent DNA sites $(b 1, b 2$, and $b 3)$ located in the rpmH-dnaA intergenic region, in the dnaA gene, and in the oriC 5' end, respectively. The authors noted that the bent DNA sites induced a curved DNA structure in those regions and are probably involved in DNA replication initiation for this organism.

Recently, numerous studies with nucleotide sequences of Archaeobacteria have revealed that replication initiation of genomic DNA contains similarities with the eukaryotic genome (Myllykallio et al., 2000; Vas and Leatherwood, 2000), but that there are no commonly found consensus tract sequences among these organisms (Aladjem and Fanning, 2004; Cvetic and Walter, 2005). These results suggest that structural motifs could be involved in the Archaeobacteria replication process.

In resume, in prokaryotic replication initiation the binding of an initiation protein at the replication origin causes a helix opening adjacent to the region containing AT-rich segments (Bramhill and Kornberg, 1988). Specific protein-binding sequences in prokaryotic replication origins can present conserved structural domains, such as curved DNA, which plays an important role in DNA replication (Eckdahl and Anderson, 1990; Hosid et al., 2004).

\section{BENT DNA SITES IN EUKARYOTIC REPLICATION ORIGIN}

During the last decade, molecular biology has made a lot of progress in the identification of initiation sites of DNA replication in eukaryotes. However, in higher eukaryotes, it 
is difficult to define the replication origins, because of the complexity of these organisms. In lower eukaryotes, replication origins are understood best, such as for Saccharomyces cerevisiae, where intrinsically bent DNA sites have been found in the autonomously replicating sequences (Marahrens and Stillman, 1992; Vernis et al., 1999); this indicates a structural element to increasing the affinity to DNA protein in AT-rich tracts (Williams et al., 1988).

Schizosaccharomyces pombe, with a similar chromosomal organization to metazoans (Robinson and Bell, 2005), has contributed to a better understanding of the structure of the replication origin for multicellular organisms. In the replication origin, nucleotide sequences of 600-800 bp have been identified that contain AT-rich tracts. These elements are larger than those of $S$. cerevisiae, and it is not possible to identify consensus sequences in the autonomously replicating sequences (Okuno et al., 1999; Kelly and Brown, 2000).

Marilley et al. (2007) found a strong intrinsic curvature in the Spars1 origin of Schizosaccharomyces pombe through electrophoresis, computational analysis, and atomic force microscopy. In this yeast, the orp4p protein was identified in the HMG1(Y) chromatin protein. This protein is a homolog of orc4 in S. cerevisiae (Chuang and Kelly, 1999). The high mobility groups are protein-binding bent DNA sites (Stros, 2001).

Du et al. (1995) have shown that restriction fragments of the 5'NTS rRNA genes' replication origin in Tetrahymena thermophila contain segments of bent DNA, suggesting that these structures may facilitate initiator protein interactions or the association of the origin with the nuclear matrix.

If the chromatin structure is more open, such as that observed at bent DNA sites, this may facilitate the complex binding of the origin recognition complex and determine the localization of initiation sites (Gerbi et al., 2002). The origin recognition complex is evolutionarily conserved; it is present in all eukaryotic cells and has six proteins, which show a relationship between structure and function (for a review, see Bell, 2002). The presence of replication factors that make up several replication sites and the association of these sites with the nuclear matrix (S/MAR) show that the chromosome structure in this process is essential (Anachkova et al., 2005; Fiorini et al., 2006b).

Gene amplification provides a useful model for studying the replication origin structure and the control of the initiation replication. The models that have been studied most involve chromosomes that contain the chorion genes of Drosophila melanogaster (Claycomb and OrrWeaver, 2005) and DNA puffs of sciarid flies, such as Rhynchosciara americana (Yokosawa et al., 1999; Soares et al., 2003). In Rhynchosciara americana, multiple bent DNA sites were found in the replication origin encompassing the C3-22 gene (Fiorini et al., 2006a).

The involvement of origins within amplified segments that include bent DNA sites was also described in mammalian cells. Caddle et al. (1990) examined the relationship between bent DNA sites and replication origins localized in the dihydrofolate reductase gene intergenic region in an amplified segment of Chinese hamster ovary culture cells. Recently, Altman and Fanning (2004) showed that an ori- $\beta$ initiation segment in the dihydrofolate reductase gene intergenic region required four specific elements for full activity of the ori- $\beta$ region, one of these was an intrinsically bent DNA site that facilitated the initiation of replication in the ori- $\beta$ site. We described intrinsically bent DNA sites near replication origins oriGNAI3 and ori $B$ in the Chinese hamster Adenylate deaminase 2 (AMPD2) amplicon (Balani et al., 2006). Coffman et al. (2006) also reported an intrinsic DNA bending site within several hundred base pairs of one initiation site present in a non-transcribed spacer region of the human ribosomal RNA gene (rDNA). 


\section{BENT DNA SITES IN PROMOTER REGIONS}

The discovery of alternative structures, such as curvature in the promoter and replication origins that were either induced by the intrinsic DNA sequence or produced by DNA flexibility, permitted the establishment of a relationship between structure and function (Zhang et al., 2004). Bent DNA sites are one of the principal structures that influence transcription and nucleosome positioning in promoter regions (Wanapirak et al., 2003).

The presence of intrinsically bent sites in promoter regions provides the necessary conditions for protein binding to DNA. The TATA-box-binding protein binds at the TATA-box region in the minor groove (Lee et al., 1991; Starr and Hawley, 1991); this may be facilitated by the curved segment that allows the transcriptional activity. Several studies have shown a correlation between the rotational orientation of the curved DNA relative to the promoter and its ability to enhance transcription (McAllister and Achberger, 1989).

Intrinsically bent DNA was found in promoter regions of the ribosomal gene in Xenopus laevis and in sequences of the intergenic region that are involved in transcription regulation (Roux-Rouquie and Marilley, 2000). In yeast, using electrophoretic analyses and circular permutation, bent DNA sites were identified in the gene promoters GAL1-10 and GAL80 regulatory genes (Bash et al., 2001).

In several eukaryotic promoters, Miyano et al. (2001) identified intrinsically bent DNA sites and proposed that those bent structures may be responsible for organizing the chromatin local conformation and for regulating the beginning of transcription. Fiorini et al. (2001) detected an intrinsically bent DNA site in the promoter region of the BhC4-1 gene in Bradysia hygida (Fiorini et al., 2001) and Reis et al. (2004) described three bent DNA sites downstream of this gene, showing that the BhC4-1 gene is flanked by bent sites. Another description for the amplified gene $A M P D 2$ in lung fibroblast culture cells of the Chinese hamster also identified bent DNA sites flanking the genes in the amplicon (Balani et al., 2006).

In Rhynchosciara americana, three bent DNA sites were found in the promoter region and in the downstream sequence of the C3-22 amplified gene (Gouveia et al., 2008). This result, along with the one described for the C3-22 amplicon replication origin (Fiorini et al., 2006a), suggest that bent DNA sites are involved in the differential replication and transcription processes of this genomic segment.

DNA curvature was also reported in the promoter region of the human $\varepsilon$-globin gene and subsequently in other regions of the same locus (Wada-Kiyama and Kiyama, 1996; Onishi et al., 1998). The presence of an intrinsic DNA bend with a broad locus of curvature for the human $c d c 2$ promoter indicates that this structure influences the interaction of regulatory factors with the transcription machinery (Nair, 1998).

\section{FINAL CONSIDERATIONS}

Regulatory regions, such as promoters and segments with replication origins in eukaryotes and prokaryotes, present alternative three-dimensional conformations induced by intrinsic bent DNA sites. It has been reported by several authors that this structure has an influence on transcriptional regulation, promoting the association with transcription factors and/ or enhancing transcription. The role of this in DNA replication is not clear, but this structure can enable helix unwinding and also allow for the interaction of proteins that participate in the 
replication machinery. Therefore, the study and mapping of intrinsic bent sites in these regions is crucial for understanding the molecular dynamics of the cell.

\section{ACKNOWLEDGMENTS}

We thank Valmir Peron and Marli Licero Schuete Silva for dedicated technical assistance. K.I. Takeda was the recipient of a fellowship from Conselho Nacional de Desenvolvimento Científico e Tecnológico (CNPq). Research supported by grants from Conselho Nacional de Desenvolvimento Científico e Tecnológico (CNPq), and the Academy of Sciences for the Developing World (TWAS).

\section{REFERENCES}

Aladjem MI and Fanning E (2004). The replicon revisited: an old model learns new tricks in metazoan chromosomes. EMBO Rep. 5: 686-691.

Altman AL and Fanning E (2004). Defined sequence modules and an architectural element cooperate to promote initiation at an ectopic mammalian chromosomal replication origin. Mol. Cell Biol. 24: 4138-4150.

Anachkova B, Djeliova V and Russev G (2005). Nuclear matrix support of DNA replication. J. Cell Biochem. 96: 951-961.

Balani VA, Takeda KI, Fiorini A and Fernandez MA (2006). Bent DNA sites in mammalian replication origins. In: XXXV Reunião Anual da SBBq, Águas de Lindóia, CD-ROM.

Barbosa JF, Bravo JP, Takeda KI, Zanatta DB, et al. (2008). Intrinsic bent DNA colocalizes with the sequence involved in the Nd-sD mutation in Fibroin light chain gene of the Bombyx mori. BMB Rep. 41: 394-399.

Bash RC, Vargason JM, Cornejo S, Ho PS, et al. (2001). Intrinsically bent DNA in the promoter regions of the yeast GAAL1-10 and GAL80 genes. J. Biol. Chem. 276: 861-866.

Bell SP (2002). The origin recognition complex: from simple origins to complex functions. Genes Dev. 16: 659-672.

Bolshoy A, McNamara P, Harrington RE and Trifonov EN (1991). Curved DNA without A-A: experimental estimation of all 16 DNA wedge angles. Proc. Natl. Acad. Sci. U. S. A. 88: 2312-2316.

Bramhill D and Kornberg A (1988). Duplex opening by dnaA protein at novel sequences in initiation of replication at the origin of the E. coli chromosome. Cell 52: 743-755.

Brylawski BP, Cohen SM, Cordeiro-Stone M, Schell MJ, et al. (2000). On the relationship of matrix association and DNA replication. Crit. Rev. Eukaryot. Gene Expr. 10: 91-99.

Caddle MS, Lussier RH and Heintz NH (1990). Intramolecular DNA triplexes, bent DNA and DNA unwinding elements in the initiation region of an amplified dihydrofolate reductase replicon. J. Mol. Biol. 211: 19-33.

Calladine CR, Drew HR, Luisi BF and Travers AA (2004). Understanding DNA: The Molecule and How it Works. 3rd edn. Academic Press, London.

Chuang RY and Kelly TJ (1999). The fission yeast homologue of Orc4p binds to replication origin DNA via multiple AThooks. Proc. Natl. Acad. Sci. U. S. A. 96: 2656-2661.

Clarey MG, Erzberger JP, Grob P, Leschziner AE, et al. (2006). Nucleotide-dependent conformational changes in the DnaA-like core of the origin recognition complex. Nat. Struct. Mol. Biol. 13: 684-690.

Claycomb JM and Orr-Weaver TL (2005). Developmental gene amplification: insights into DNA replication and gene expression. Trends Genet. 21: 149-162.

Coffman FD, He M, Diaz ML and Cohen S (2006). Multiple initiation sites within the human ribosomal RNA gene. Cell Cycle 5: 1223-1233.

Cvetic C and Walter JC (2005). Eukaryotic origins of DNA replication: could you please be more specific? Semin. Cell Dev. Biol. 16: 343-353.

Du C, Sanzgiri RP, Shaiu WL, Choi JK, et al. (1995). Modular structural elements in the replication origin region of Tetrahymena rDNA. Nucleic Acids Res. 23: 1766-1774.

Eckdahl TT and Anderson JN (1987). Computer modelling of DNA structures involved in chromosome maintenance. Nucleic Acids Res. 15: 8531-8545.

Eckdahl TT and Anderson JN (1990). Conserved DNA structures in origins of replication. Nucleic Acids Res. 18: 1609-1612. 
Erzberger JP, Mott ML and Berger JM (2006). Structural basis for ATP-dependent DnaA assembly and replication-origin remodeling. Nat. Struct. Mol. Biol. 13: 676-683.

Fiorini A, Basso LR Jr, Paco-Larson ML and Fernandez MA (2001). Mapping of intrinsic bent DNA sites in the upstream region of DNA puff BhC4-1 amplified gene. J. Cell Biochem. 83: 1-13.

Fiorini A, de Gouveia FS, de Soares MA, Stocker AJ, et al. (2006a). DNA bending in the replication zone of the C3 DNA puff amplicon of Rhynchosciara americana (Diptera: Sciaridae). Mol. Biol. Rep. 33: 71-82.

Fiorini A, Gouveia FS and Fernandez MA (2006b). Scaffold/matrix attachment regions and intrinsic DNA curvature. Biochemistry 71: 481-488.

Fujimura FK (1988). Point mutation in the polyomavirus enhancer alters local DNA conformation. Nucleic Acids Res. 16: 1987-1997.

Gerbi SA, Strezoska Z and Waggener JM (2002). Initiation of DNA replication in multicellular eukaryotes. J. Struct. Biol. 140: $17-30$.

Gimenes F, Gouveia FS, Fiorini A and Fernandez MA (2008). Intrinsic bent DNA sites in the chromosomal replication origin of Xylella fastidiosa 9a5c. Braz. J. Med. Biol. Res. 41: 295-304.

Gouveia FS, Gimenes F, Fiorini A and Fernandez MA (2008). Intrinsic bent DNA sites in the developmentally amplified C3-22 gene promoter of Rhynchosciara americana (Diptera: Sciaridae). Biosci. Biotechnol. Biochem. 72: 1190-1198.

Haran TE and Crothers DM (1989). Cooperativity in A-tract structure and bending properties of composite TnAn blocks. Biochemistry 28: 2763-2767.

Hosid S, Trifonov EN and Bolshoy A (2004). Sequence periodicity of Escherichia coli is concentrated in intergenic regions. BMC Mol. Biol. 5: 14.

Iarovaia OV, Borounova V, Vassetzky YS and Razin SV (2006). An unusual extended DNA loop attachment region is located in the human dystrophin gene. J. Cell Physiol. 209: 515-521.

Kelly TJ and Brown GW (2000). Regulation of chromosome replication. Annu. Rev. Biochem. 69: 829-880.

Kim J, Zwieb C, Wu C and Adhya S (1989). Bending of DNA by gene-regulatory proteins: construction and use of a DNA bending vector. Gene $85: 15-23$.

Klungsoyr HK and Skarstad K (2004). Positive supercoiling is generated in the presence of Escherichia coli SeqA protein. Mol. Microbiol. 54: 123-131.

Lee DK, Horikoshi M and Roeder RG (1991). Interaction of TFIID in the minor groove of the TATA element. Cell 67: 1241-1250.

Levene SD and Crothers DM (1983). A computer graphics study of sequence-directed bending in DNA. J. Biomol. Struct. Dyn. 1: 429-435.

Marahrens Y and Stillman B (1992). A yeast chromosomal origin of DNA replication defined by multiple functional elements. Science 255: 817-823.

Marilley M and Pasero P (1996). Common DNA structural features exhibited by eukaryotic ribosomal gene promoters. Nucleic Acids Res. 24: 2204-2211.

Marilley M, Milani P, Thimonier J, Rocca-Serra J, et al. (2007). Atomic force microscopy of DNA in solution and DNA modelling show that structural properties specify the eukaryotic replication initiation site. Nucleic Acids Res. 35: $6832-6845$.

McAllister CF and Achberger EC (1989). Rotational orientation of upstream curved DNA affects promoter function in Bacillus subtilis. J. Biol. Chem. 264: 10451-10456.

Messer W (2002). The bacterial replication initiator DnaA. DnaA and oriC, the bacterial mode to initiate DNA replication. FEMS Microbiol. Rev. 26: 355-374.

Milot E, Belmaaza A, Wallenburg JC, Gusew N, et al. (1992). Chromosomal illegitimate recombination in mammalian cells is associated with intrinsically bent DNA elements. EMBO J. 11: 5063-5070.

Miyano M, Kawashima T and Ohyama T (2001). A common feature shared by bent DNA structures locating in the eukaryotic promoter region. Mol. Biol. Rep. 28: 53-61.

Monteiro PB, Teixeira DC, Palma RR, Garnier M, et al. (2001). Stable transformation of the Xylella fastidiosa citrus variegated chlorosis strain with oriC plasmids. Appl. Environ. Microbiol. 67: 2263-2269.

Myllykallio H, Lopez P, Lopez-Garcia P, Heilig R, et al. (2000). Bacterial mode of replication with eukaryotic-like machinery in a hyperthermophilic archaeon. Science 288: 2212-2215.

Nair TM (1998). Evidence for intrinsic DNA bends within the human cdc2 promoter. FEBS Lett. 422: 94-98.

Okuno Y, Satoh H, Sekiguchi M and Masukata H (1999). Clustered adenine/thymine stretches are essential for function of a fission yeast replication origin. Mol. Cell Biol. 19: 6699-6709.

Onishi Y, Wada-Kiyama Y and Kiyama R (1998). Expression-dependent perturbation of nucleosomal phases at HS2 of the human beta-LCR: possible correlation with periodic bent DNA. J. Mol. Biol. 284: 989-1004. 
Palin AH, Critcher R, Fitzgerald DJ, Anderson JN, et al. (1998). Direct cloning and analysis of DNA sequences from a region of the Chinese hamster genome associated with aphidicolin-sensitive fragility. J. Cell Sci. 111 (Pt 12): 1623-1634.

Pasero P, Sjakste N, Blettry C, Got C, et al. (1993). Long-range organization and sequence-directed curvature of Xenopus laevis satellite 1 DNA. Nucleic Acids Res. 21: 4703-4710.

Reis TF, Fiorini A and Fernandez MA (2004). Estudo de sítios de DNA bent na região à jusante do gene amplificado BhC4-1 de Bradysia hygida. In: 50 Congresso Brasileiro de Genética, Florianópolis, Abstract GA, 179.

Remus D, Beall EL and Botchan MR (2004). DNA topology, not DNA sequence, is a critical determinant for Drosophila ORC-DNA binding. EMBO J. 23: 897-907.

Robinson NP and Bell SD (2005). Origins of DNA replication in the three domains of life. FEBS J. 272: 3757-3766.

Roux-Rouquie M and Marilley M (2000). Modeling of DNA local parameters predicts encrypted architectural motifs in Xenopus laevis ribosomal gene promoter. Nucleic Acids Res. 28: 3433-3441.

Soares MA, Monesi N, Basso LR Jr, Stocker AJ, et al. (2003). Analysis of the amplification and transcription of the C3-22 gene of Rhynchosciara americana (Diptera: Sciaridae) in transgenic lines of Drosophila melanogaster. Chromosoma 112: 144-151.

Sperbeck SJ and Wistow GJ (1998). pBendBlue: modification of the pBend system for color selectability. Biotechniques 24: 66-68.

Starr DB and Hawley DK (1991). TFIID binds in the minor groove of the TATA box. Cell 67: 1231-1240.

Stros M (2001). Two mutations of basic residues within the N-terminus of HMG-1 B domain with different effects on DNA supercoiling and binding to bent DNA. Biochemistry 40: 4769-4779.

Trifonov EN and Sussman JL (1980). The pitch of chromatin DNA is reflected in its nucleotide sequence. Proc. Natl. Acad. Sci. U. S. A. 77: 3816-3820.

Vas A and Leatherwood J (2000). Where does DNA replication start in archaea? Genome Biol. 1: REVIEWS1020.

Vernis L, Chasles M, Pasero P, Lepingle A, et al. (1999). Short DNA fragments without sequence similarity are initiation sites for replication in the chromosome of the yeast Yarrowia lipolytica. Mol. Biol. Cell 10: 757-769.

Virstedt J, Berge T, Henderson RM, Waring MJ, et al. (2004). The influence of DNA stiffness upon nucleosome formation. J. Struct. Biol. 148: 66-85.

Wada-Kiyama Y and Kiyama R (1996). Conservation and periodicity of DNA bend sites in eukaryotic genomes. DNA Res. 3: 25-30.

Wanapirak C, Kato M, Onishi Y, Wada-Kiyama Y, et al. (2003). Evolutionary conservation and functional synergism of curved DNA at the mouse epsilon- and other globin-gene promoters. J. Mol. Evol. 56: 649-657.

Wang J and Sugden B (2005). Origins of bidirectional replication of Epstein-Barr virus: models for understanding mammalian origins of DNA synthesis. J. Cell Biochem. 94: 247-256.

Williams JS, Eckdahl TT and Anderson JN (1988). Bent DNA functions as a replication enhancer in Saccharomyces cerevisiae. Mol. Cell Biol. 8: 2763-2769.

Wu HM and Crothers DM (1984). The locus of sequence-directed and protein-induced DNA bending. Nature 308: 509-513.

Yokosawa J, Soares MA, Dijkwel PA, Stocker AJ, et al. (1999). DNA replication during amplification of the C3 puff of Rhynchosciara americana initiates at multiple sites in a $6 \mathrm{~kb}$ region. Chromosoma 108: 291-301.

Young MA and Beveridge DL (1998). Molecular dynamics simulations of an oligonucleotide duplex with adenine tracts phased by a full helix turn. J. Mol. Biol. 281: 675-687.

Zhang Y, Xi Z, Hegde RS, Shakked Z, et al. (2004). Predicting indirect readout effects in protein-DNA interactions. Proc. Natl. Acad. Sci. U. S. A. 101: 8337-8341. 